\title{
Fracture Mechanics Analysis of LH2 Feed Line Flow Liners
}

\author{
Mark A. James ${ }^{1}$, David S. Dawicke ${ }^{2}$, Matthew B. Brzowski ${ }^{3}$, \\ Ivatury S. Raju ${ }^{4}$, Kenny B. Elliott, and Charles E. Harris ${ }^{5}$ \\ NASA Langley Research Center \\ Hampton VA 23681
}

\begin{abstract}
Inspections of the Space Shuttle Main Engine revealed fatigue cracks growing from slots in the flow liner of the liquid hydrogen (LH2) feed lines. During flight, the flow liners experience complex loading induced by flow of LH2 and the resonance characteristics of the structure. The flow liners are made of Inconel 718 and had previously not been considered a fracture critical component. However, fatigue failure of a flow liner could have catastrophic effect on the Shuttle engines.

A fracture mechanics study was performed to determine if a damage tolerance approach to life management was possible and to determine the sensitivity to the load spectra, material properties, and crack size. The load spectra were derived separately from ground tests and material properties were obtained from coupon tests. The stress-intensity factors for the fatigue cracks were determined from a shell-dynamics approach that simulated the dominant resonant frequencies. Life predictions were obtained using the NASGRO life prediction code. The results indicated that adequate life could not be demonstrated for initial crack lengths of the size that could be detected by traditional NDE techniques.
\end{abstract}

\section{Introduction}

During an inspection of the Space Shuttle Main Engine, fatigue cracks were found in the flow liner of the liquid hydrogen feed line. The flow liner was designed as a non-structural member that is used to maintain laminar flow of the fuel in the feed line and has not been considered to be fracture critical. Because the flow liner is not a structural member, it became apparent that the loading that initiated and propagated the fatigue cracks was induced by the complex flow physics of the liquid hydrogen interacting with the resonant characteristics of the flow liner. As a result, the analysis of the crack growth behavior required a multi-disciplinary approach that derived input from a variety of sources, including flow physics, dynamics, existing and new experimental results, destructive and non-destructive evaluation results, and existing and new fracture mechanics analyses. Detailed investigations and laboratory testing indicated that the vibration could be characterized by several dominant resonant frequencies, one during each of the various major stages of launch and flight into orbit [1].

\footnotetext{
${ }^{1}$ Presently, ALCOA Technical Center, ALCOA Center, PA 15069

${ }^{2}$ Analytical Services and Materials, Inc., Hampton, VA 23666

${ }^{3}$ Presently, Lockheed Martin Commercial Space Systems, Newton, PA 18940

${ }^{4}$ Fellow, AIAA

${ }^{5}$ Associate Fellow, AIAA
} 
This paper describes a fracture mechanics-based evaluation that was undertaken to determine if a damage tolerance approach to life management was possible and to determine sensitivity to loads, material properties, and crack size. The approach used was to develop a fracture mechanics-based stress-intensity solution for the various crack growth scenarios. Then, the loading derived in cooperation with the flow physics and dynamics teams was applied. Life predictions were made using the fracture mechanics software NASGRO [2]. Several initial crack sizes were considered, and for each of these crack sizes, the life prediction calculations were performed. Several ranges of the magnitude of welding residual stresses, material crack growth rate characteristics, and other salient variables were also considered. For each of these combinations, the life prediction calculations were performed to provide insight into flight safety, inspection intervals, and inspection criteria.

\section{Flow Liner Configurations}

The upstream liner is a cylindrical shell and the downstream liner is a doubly curved cylindrical shell; both are about 12 inches in diameter and about 3 inches wide by about 0.05 inches thick, (see Figure 1). The liners are each welded at opposite ends (as indicated by the green hash marks of Figure 1) of the main structure at a joint in the feed line, and the liners overlap in the middle to maintain the laminar flow through the joint. Each flow liner has slots oriented in the direction of the flow. Fatigue cracks initiated and propagated from the slots both axially at locations A and D and circumferentially at locations B and C in Figure 2.

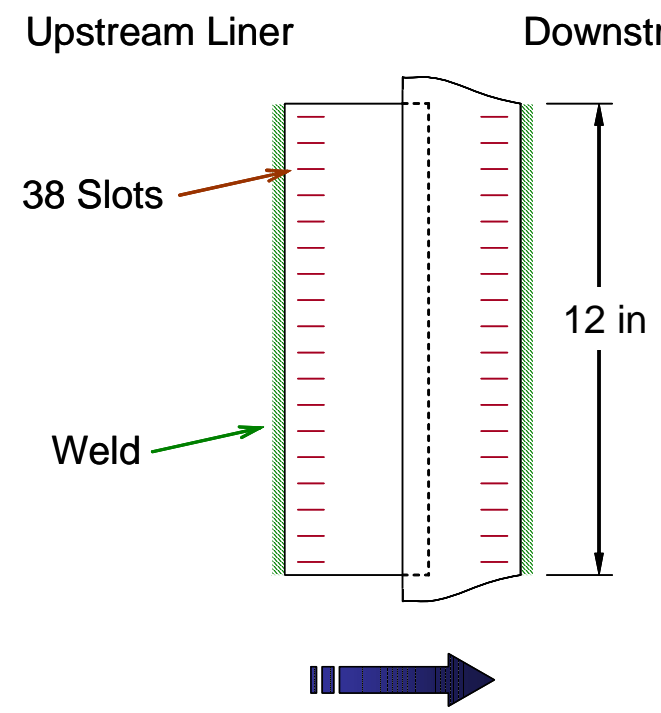

Flow Direction

Figure 1. Upstream and downstream flow liners

Cracks that initiate at locations $\mathrm{A}$ and $\mathrm{D}$ have been shown to be self limiting [1]. At location A, the crack is growing from the thin sheet liner into the thick structure that is near the weld, and thus the driving force is reduced. However, the residual stresses are highest at this location 
because of its proximity to the weld. For location D, detailed shell finite element analyses showed that crack growth away from a slot towards the edge of the liner initially increases, but then decreases because the structure resonance changes with crack growth. The two circumferential locations, locations B and C, have approximately the same stresses, but location $\mathrm{B}$ has a higher residual stress because it is closer to the weld. The current investigation selected location B for detailed examination because the residual stresses make location B more critical than location $\mathrm{C}$, and the crack growth does not appear to be self limiting like at locations A and D.

Failure of a circumferential crack in a liner occurs when the crack has grown across the entire ligament (a length of 0.75 in.). A completely cracked ligament can form a tab that can break off and get ingested into the engine. Such an ingestion can cause catastrophic damage to the engine and the Shuttle. The current analysis defines failure when the circumferential crack grows to a length of $0.6 \mathrm{in}$. This is a slightly more conservative assumption than the crack growth across the complete ligament length of 0.75 in. (The difference in lives for a crack length of 0.6 in. in comparison to 0.75 in. is shown to be negligible [1].)

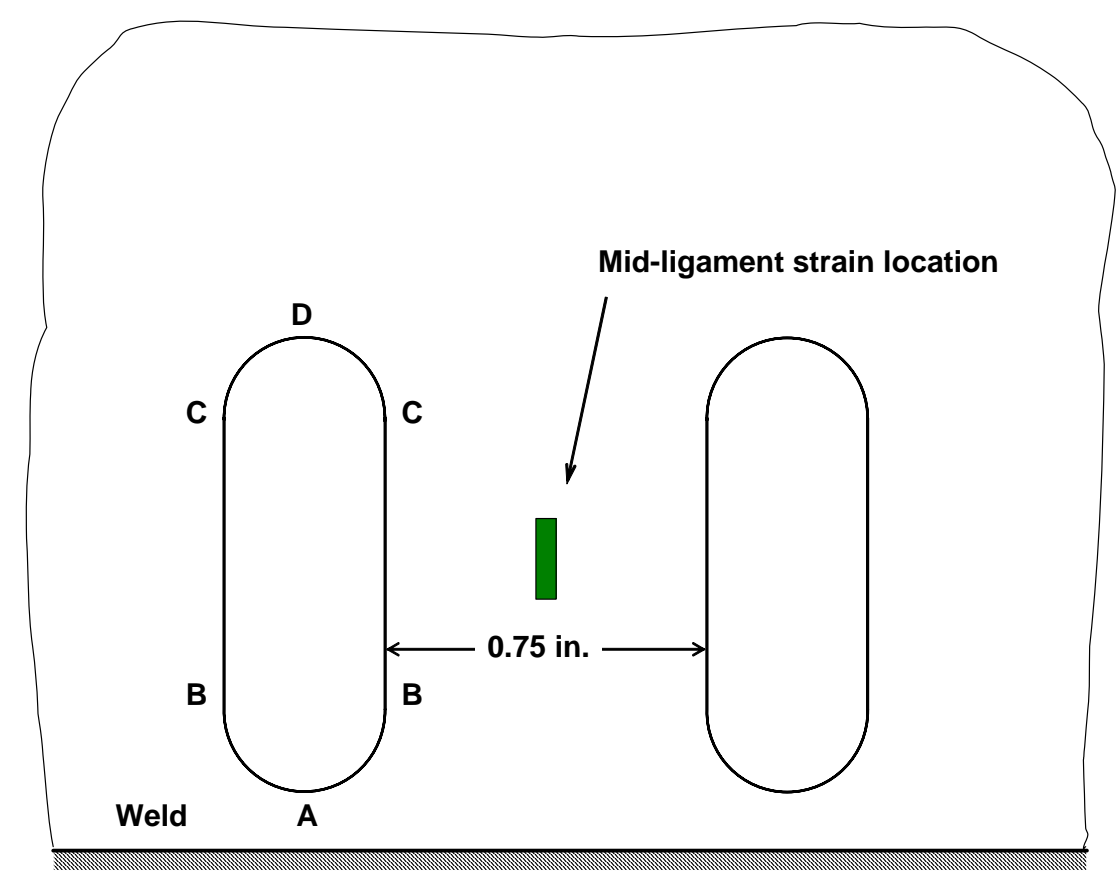

Figure 2. Schematic of cracking locations around a typical slot and location of strain measurement

\section{Loading}

The flow liners are subjected to complex loading due to the resonant response to the liquid hydrogen flow field. The computational fluid dynamics (CFD) and the flow-physics teams investigated large scale unsteady motions of the mean flow, back flow, and changes in the acoustic modes. The loads and the dynamics team, utilizing flow tests performed at Stennis Space Center [1] intended to simulate flight conditions, as well as flow-physics/CFD results, identified a predominant $3500 \mathrm{~Hz}$ complex 9ND mode (complex here refers to both membrane 
and bending modes acting simultaneously) for the upstream liner and 3ND $(1650 \mathrm{~Hz}), \mathrm{C} 4 \mathrm{ND}$ $(3300 \mathrm{~Hz})$, and $5 \mathrm{ND}(1070 \mathrm{~Hz})$ modes for the downstream liners. ( $j \mathrm{ND}$ here refers to the $j$-nodal diameter mode shape and $\mathrm{C}$ denotes complex mode shape). Based on the test data and analytical results, fatigue-loading spectra were developed [1] to simulate the loads experienced during engine operation in one flight. (In one flight the engines run for about 500 seconds). These spectra are used in the current fracture mechanics analyses.

The loading spectra are based on strains measured in the flow liner test article at the mid ligament locations between the slots (see Figure 2) during tests performed at Stennis Space Center [1]. These strains are used as scale factors on the loading spectra to evaluate the stressintensity factors. A high level of uncertainty exists in the magnitude and sequence of the flight spectra due to the complexity of the flow field and reliance on ground simulations. The details of this approach were described in Reference 1.

\section{Life Prediction Modeling}

The life prediction code NASGRO Version 4.11 [2] is used for all crack growth predictions. The stress-intensity factors are entered using a 1-D data table (DT01) option. The user dimension, D, is $0.75 \mathrm{in}$. (the width between the slots). The loading spectra are entered as separate load cases for mean bending, alternating bending, and alternating membrane. The load cases are superimposed in NASGRO during the life calculations. All life calculations are performed using the NASGRO non-interaction model to ensure the most conservative life calculations. The noninteraction model performs linear accumulated damage crack growth and deactivates plasticity induced retardation models.

Load ratio (R) effects, such as plasticity or roughness-induced crack closure, are extrinsic effects that decrease the crack growth rate by reducing the amount of damage caused by the cyclic loading and thus extend life. These effects are strongly dependent on the order and magnitude of loads in the flight spectra. The inclusion of crack closure into a life prediction analysis will produce less conservative results and cannot be justified when the uncertainty of load spectra is high. The loading used in this analysis had a high level of uncertainty, thus the load ratio effects were excluded from the life analysis.

For positive load ratios, the crack growth rate relationship is described by a modified form of the NASGRO equation that results from enabling the load ratio bypass option:

$$
\frac{d a}{d N}=\frac{C \Delta K^{n}\left(1-\frac{\Delta K_{\text {th }}}{\Delta K}\right)^{p}}{\left(1-\frac{K_{\max }}{K_{c}}\right)^{q}}, \quad(\mathrm{R} \geq 0)
$$


Where $\Delta \mathrm{K}$ is the stress-intensity factor range, $\mathrm{K}_{\max }$ is the maximum stress-intensity factor, $\mathrm{K}_{\mathrm{c}}$ is the fracture toughness, $\Delta \mathrm{K}_{\mathrm{th}}$ is the threshold stress-intensity factor range, and $\mathrm{C}, \mathrm{n}, \mathrm{p}$, and $\mathrm{q}$ are curve fit (Paris-like) parameters [2]. In Eq. (1), the entire $\Delta \mathrm{K}$ range contributes to crack growth. The $\Delta \mathrm{K}_{\mathrm{th}}$ term is still a function of $\mathrm{R}$ and allows Eq. (1) to fit the high $\mathrm{R}$ data near threshold.

For negative load ratios, the NASGRO equation reduces to:

$$
\frac{d a}{d N}=\frac{C \Delta K_{\max }^{n}\left(1-\frac{\Delta K_{t h}}{\Delta K}\right)^{p}}{\left(1-\frac{K_{\max }}{K_{c}}\right)^{q}} \quad(\mathrm{R}<0)
$$

Thus, for negative load ratios, only the tensile part of the load cycle is active and the crack is assumed to be closed during the compressive part of the load cycle. The loading of the flow liners is predominantly high mean stress loading with an alternating component that is smaller than the mean stress. Thus, Eq. (2) will have an insignificant influence on the NASGRO life predictions for the flow liner loading spectra.

\section{Material and Material Model}

The flow liners are constructed using Inconel 718 and operate at a temperature of $-423^{\circ} \mathrm{F}$. The material data used for the analyses was generated using liquid helium (LHe @ -423 $\mathrm{F}$ ) by researchers and engineers at NASA Marshall Space Flight Center. The liquid helium test was performed using a temperature controlled spray technique that allowed simulation of a liquid hydrogen temperature using the inert helium. Tests were performed to characterize the closure free (at or above high load ratios of $\mathrm{R}=0.7$ ) intrinsic fatigue crack growth response over a wide range of rates from threshold to fracture. Tests were also performed to characterize the low load ratio $(\mathrm{R}=0.1)$ in the Paris regime to establish the effect of plasticity induced closure; however, as mentioned above, only the high load ratio results were used in the life calculations.

The loading for the orbiter flow liner is approximated by spectra developed from flow tests intended to simulate flight conditions; however, a high level of uncertainty exists for the actual loading. The fracture mechanics-based life predictions rely on accurate loads, and in particular, an accurate description of the load ratio $\mathrm{R}$ (the ratio of minimum to maximum load). High load ratio crack growth rate data describes intrinsic material behavior. Low load ratio crack growth rate data describes material behavior that is affected by extrinsic effects such as plasticity induced crack closure. These extrinsic effects can have a significant influence on crack growth rate, thus on the life predictions as well. For example, $\mathrm{R}=0.1$ data commonly has crack growth rates that are a factor of 5 lower than $R=0.9$ data at the same value of $\Delta K$. The inclusion of load ratio effects could increase the calculated fatigue life (making predictions less conservative) in a manner that cannot be supported due to uncertainties in the assumed loading. Thus, the crack growth rate behavior for all load ratios is forced to coincide with the $\mathrm{R}=0.9$ crack growth rate curve approaching threshold. 
Figure 3 is a schematic of the material model for the crack growth rate data. The two NASGRO parameters, $S_{\max } /$ Flow $=1$ and Alpha $=5.845$, are used to minimize the load ratio effect. This is referred to as the NASGRO load ratio bypass option. Three additional NASGRO parameters, $p$, $D K 1$, and $C_{t h}$, control the fit to the high load ratio data in the threshold regime. The curves shown in Figure 3 exhibit no load ratio effect at threshold. In addition, the ' $C_{t h}$ value option' was set to 'mat'l file value throughout' to enforce that the fit to the high load ratio data was consistent throughout the analysis. The $\mathrm{K}_{\max }$ (near-fracture) behavior was allowed to maintain the load ratio influences, as indicated by the separation of the curves at large $\Delta \mathrm{K}$ values, as in Figure 3.

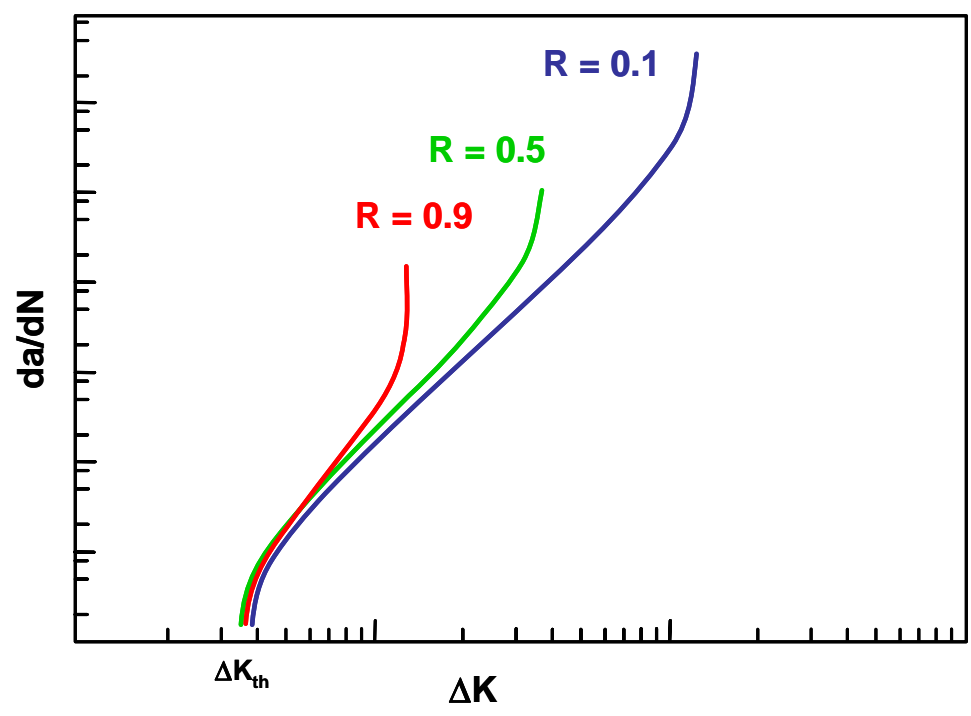

Figure 3. Notational material model for the crack growth rate data

\section{Threshold and Crack Size Considerations}

Experimental evidence from ground test articles indicate that the flow liner cracks initiate as corner cracks from surface defects that are of the size of the material microstructural features (e.g., grain size). Linear elastic fracture mechanics (LEFM) may not be applicable for cracks that are small relative to the material microstructure. Two fundamental limitations for small crack modeling are: (1) microstructurally and mechanically small cracks cannot be represented by simple continuum LEFM crack models, and (2) microstructurally small cracks may have different threshold behavior than the long crack material data available for the material models. To overcome the first of these limitations, the crack size must be large enough that the crack can be approximated as a continuum crack. A continuum crack has a cyclic plastic zone that is small compared to the length of the crack, but large relative to the size of the microstructural features. A crack that has a length greater than 10 grain sizes can generally be considered a continuum crack. Inconel 718 has reported grain sizes of $5-40 \mu \mathrm{m}(0.0002-0.0015$ in.) [1]. This grain size would require that a crack be $50-400 \mu \mathrm{m}(0.002-0.015 \mathrm{in}$.) long to be considered a continuum crack. Therefore, the analyses consider cracks larger than 0.02 in. to maintain LEFM applicability. 


\section{Approach for Stress-Intensity Factors}

A shell dynamics-based approach is used in the evaluation of stress-intensity factors. In this approach, a modal dynamic analysis of an uncracked shell model of a flow liner is performed. The representative mode of excitation in the shell is identified, a crack is introduced into the model, and the strain energy release rates at the crack tip are calculated using the eigenvector of the corresponding shell mode. The stress-intensity factors are then evaluated from the strain energy release rates using the deformed mode shapes that the liner experiences.

A typical shell finite element model of the upstream liner is shown in Figure 4. Using this model, a modal analysis is performed to isolate the dominant mode shapes (i.e. C9ND for the upstream liner). The deformed shape of the upstream liner, based on the eigenvector corresponding to this mode shape, is shown in Figure 5. Typical values of the normalized axial stress at the mid-ligament locations are plotted in Figure 6 for all of the ligaments an uncracked flow liner. As expected, the distribution shows a certain amount of cyclic symmetry for the C9ND mode shape. Similarly, the axial stresses at location B in all the slots are examined and the slot with the highest stress at location B is isolated. (There may be more than one slot with the same peak stress. In such a case, any one of those slots is chosen). The slot with the highest axial stress at location B is at $\varphi=340^{\circ}$. A circumferential crack is introduced at this slot, as shown schematically in Figure 7, and a new shell finite element model with the crack is developed and re-analyzed.
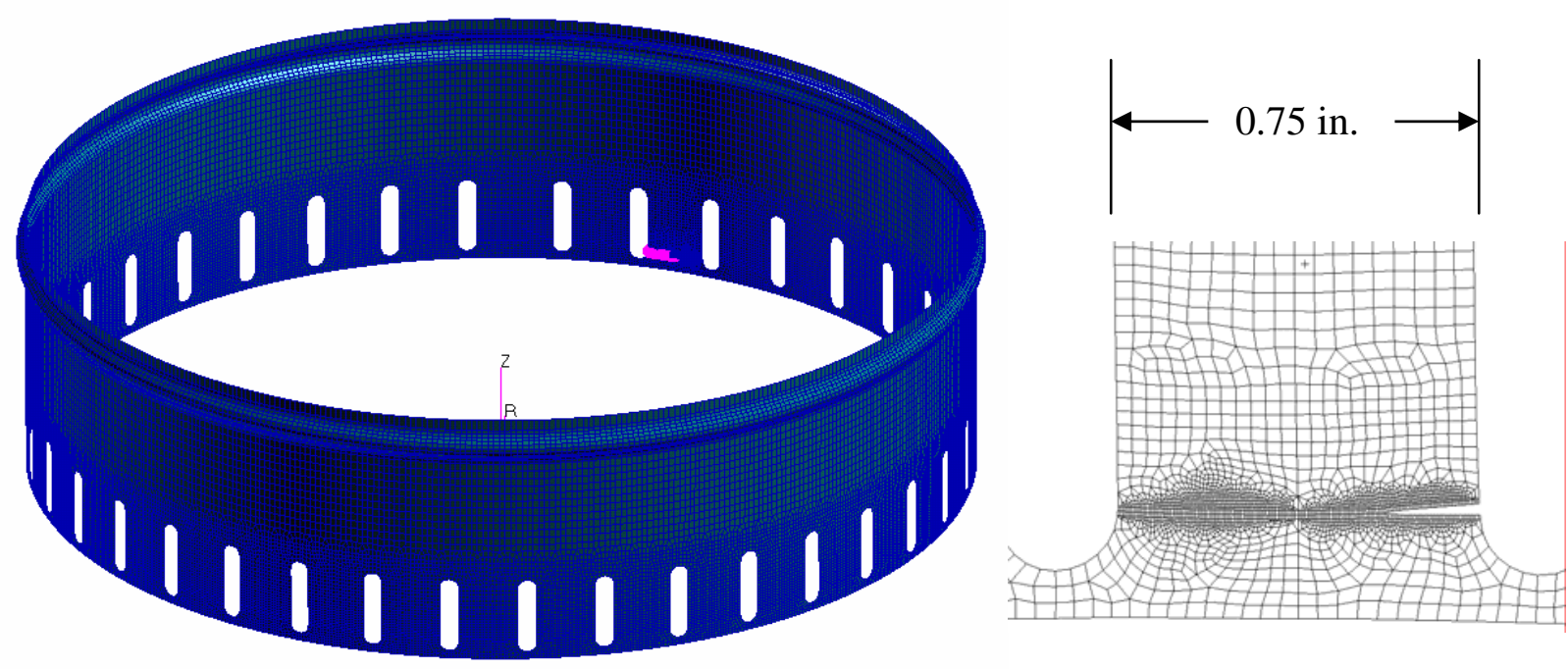

(a) Typical full liner model

(b) Typical refined mesh near the crack

Figure 4. Typical shell finite element model 

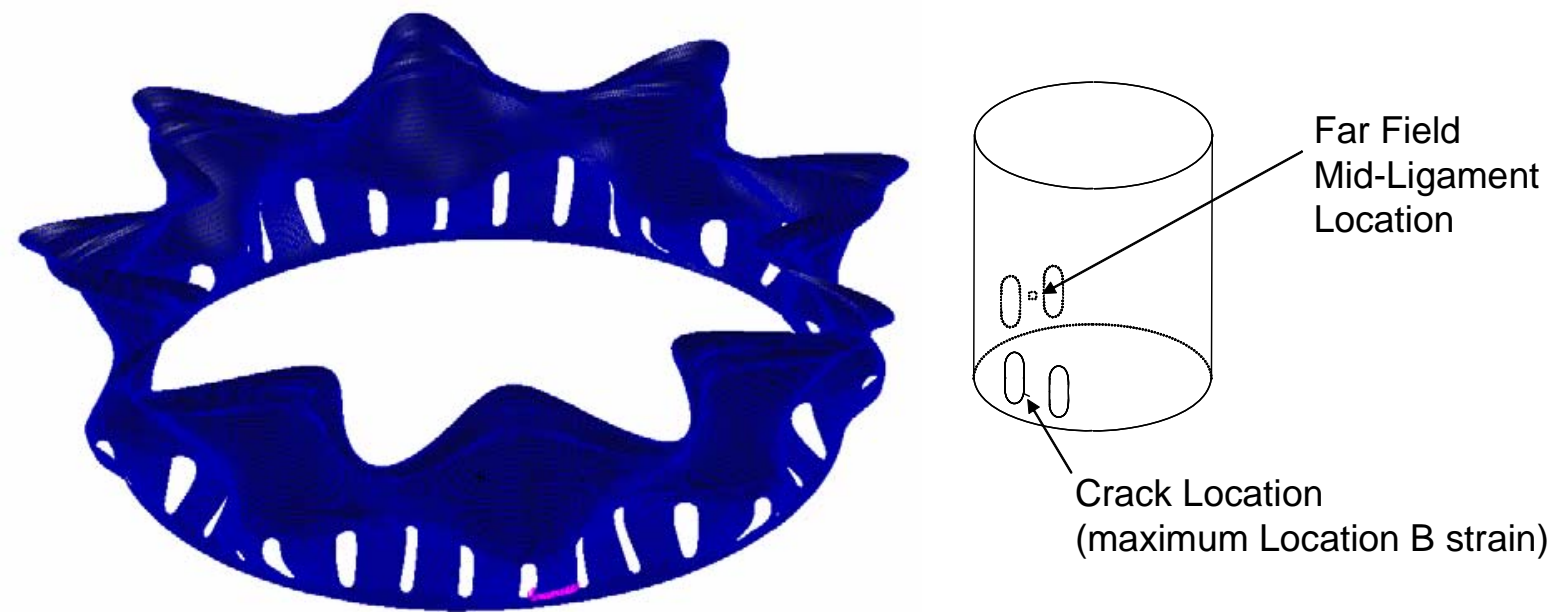

Figure 5. Far-field Ligament for Scaling the Eigen-Value Results

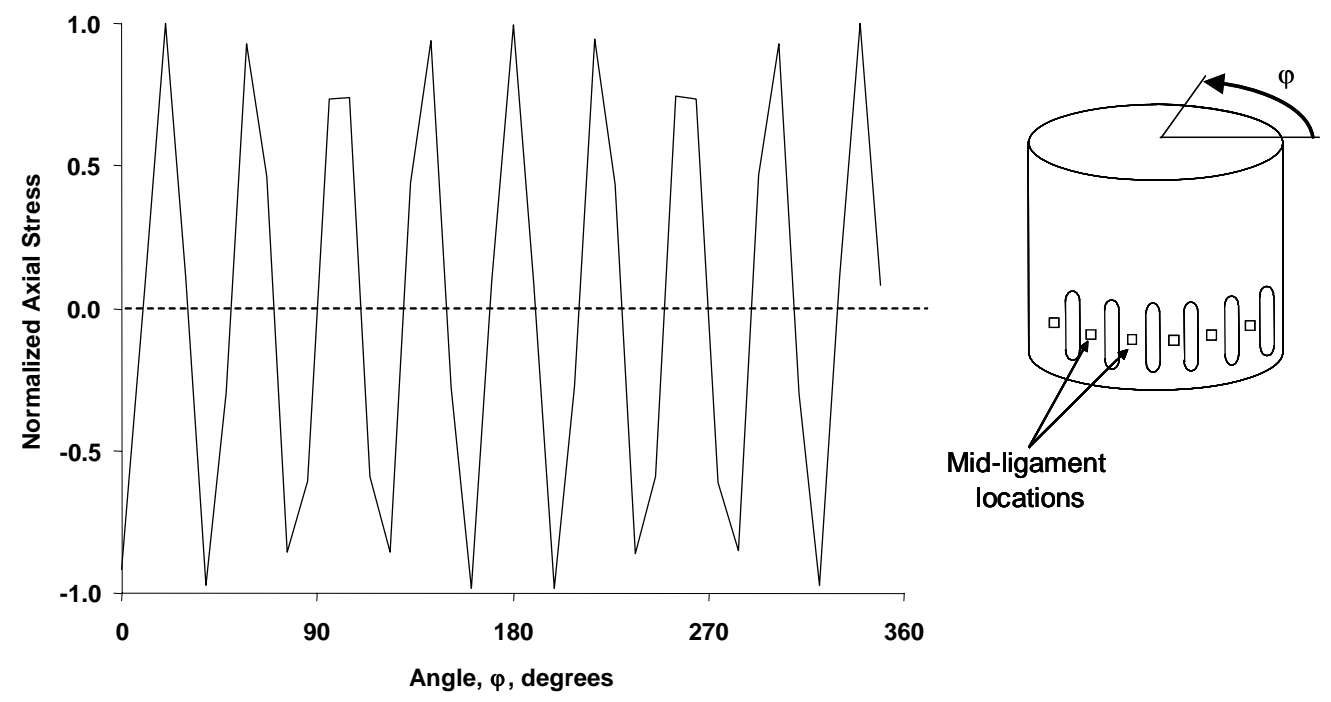

Figure 6. Typical normalized axial mid-ligament stress for the C9ND mode shape

The mode shape corresponding to the C9ND mode is isolated for the new model with the crack. The quantifiable values of the liner deformations are obtained by scaling the eigenvector with the strain gage data collected in previous ground flow liner tests [1]. The scaling process matches the maximum mid-ligament strain to strain gage measurements made at the same location. The C9ND analysis found three mid-ligament locations with nearly the same peak value, so the scaling is based on the one that is farthest $\left(\varphi=180^{\circ}\right)$ from the slot with the crack. This location is used to scale all deformations and forces for each crack length analyzed. The process is repeated for the downstream liner. 

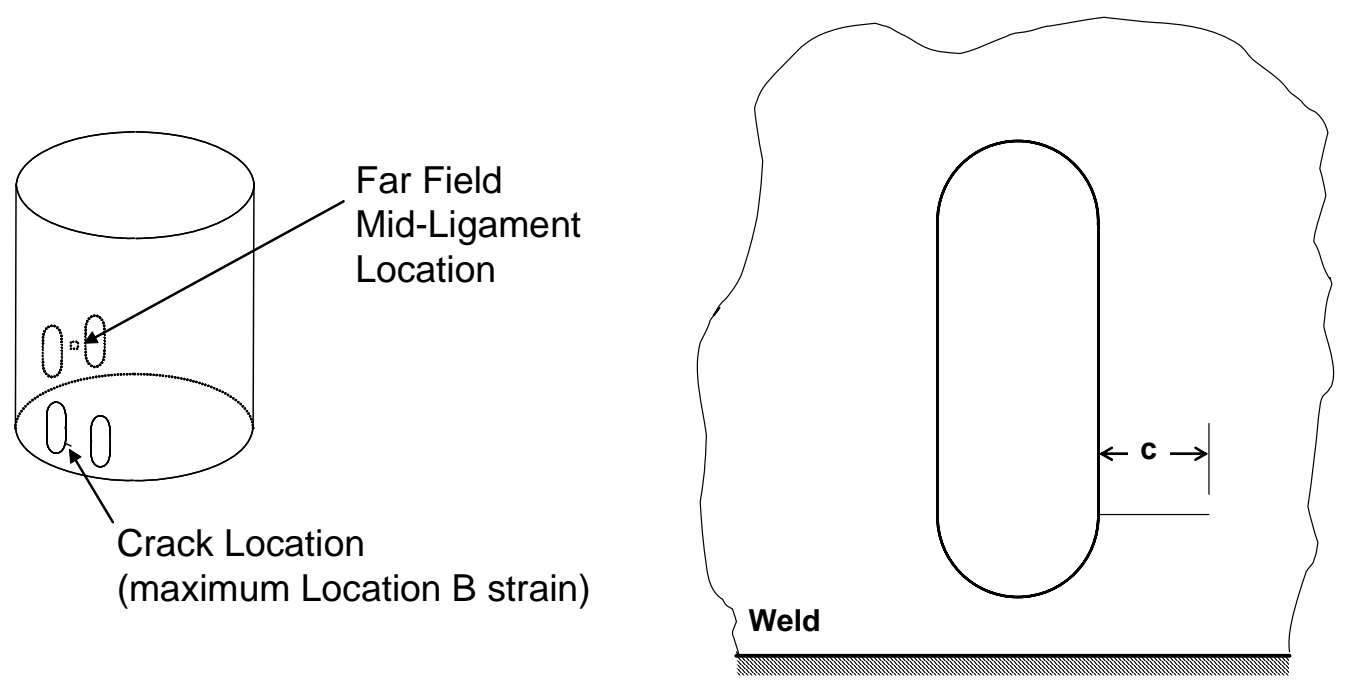

Figure 7. Schematic showing the circumferential crack at the slot with the highest location B stress

\section{Stress-intensity Factors}

The stress-intensity factors are calculated from the strain energy release rates using virtual crack closure techniques, as shown in Figure $8[5,6] . F_{x}, F_{y}$, and $F_{z}$ are the respective forces at the crack-tip node in the $\mathrm{x}-, \mathrm{y}-$, and $\mathrm{z}$-directions; $\mathrm{M}_{\mathrm{x}}, \mathrm{M}_{\mathrm{y}}$, and $\mathrm{M}_{\mathrm{z}}$ are the respective moments about the $\mathrm{x}-, \mathrm{y}-$, and $\mathrm{z}$-directions; $\mathrm{u}_{\mathrm{x}}, \mathrm{u}_{\mathrm{y}}$, and $\mathrm{u}_{\mathrm{z}}$ are the respective displacements at a node behind the crack-tip along the $\mathrm{x}-, \mathrm{y}-$, and $\mathrm{z}$-directions; and $\theta_{\mathrm{x}}, \theta_{\mathrm{y}}$, and $\theta_{\mathrm{z}}$ are the respective rotations at a node behind the crack about the $\mathrm{x}-, \mathrm{y}$-, and $\mathrm{z}$-directions. $\mathrm{t}$ is the thickness of the shell and $\Delta \mathrm{c}$ is the length of the element behind the crack tip. The finite element models have a fine mesh in the crack region with elements of the same size both behind and ahead of the crack tip (element size $\Delta \mathrm{c}=0.005$ in, as shown in Figures 4 and 8).

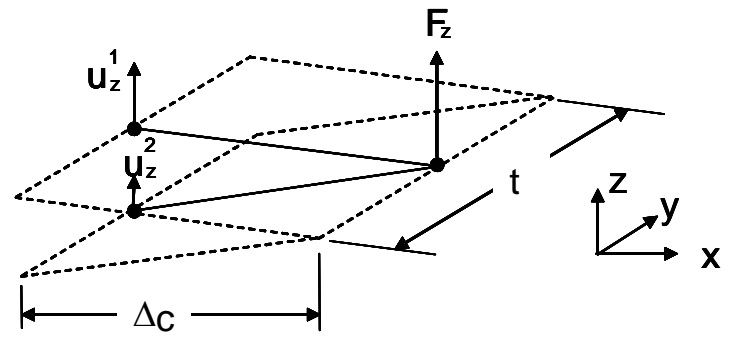

$$
\begin{aligned}
& G_{1}=\frac{1}{2 \Delta c t}\left[F_{z}\left(u_{z}^{1}-u_{z}^{2}\right)+M_{y}\left(\theta_{y}^{1}-\theta_{y}^{2}\right)+M_{x}\left(\theta_{x}^{1}-\theta_{x}^{2}\right)\right] \\
& G_{I I}=\frac{1}{2 \Delta c t}\left[F_{x}\left(u_{x}^{1}-u_{x}^{2}\right)\right] \\
& G_{I I I}=\frac{1}{2 \Delta c t}\left[F_{y}\left(u_{y}^{1}-u_{y}^{2}\right)+M_{y}\left(\theta_{2}^{1}-\theta_{z}^{2}\right)\right]
\end{aligned}
$$

Figure 8. Schematic showing crack tip coordinate system and energy release rate equations [6]

The individual mode stress-intensity factors are calculated from the energy release rates as

$$
\begin{aligned}
\mathrm{K}_{\mathrm{I}}=\sqrt{E G_{I}} \\
\mathrm{~K}_{\mathrm{II}}=\sqrt{E G_{I I}}
\end{aligned}
$$




$$
\mathrm{K}_{\mathrm{III}}=\sqrt{E G_{I I I}}
$$

where E is the Young's modulus.

In addition, a total stress-intensity factor is calculated from the total energy release rate.

$$
\mathrm{K}_{\mathrm{TOTAL}}=\sqrt{E\left(G_{I}+G_{I I}+G_{I I I}\right)}
$$

Figure 9 presents stress-intensity factor as a function of crack length calculated for the single active mode (C9ND) in the upstream liner. For each crack length, the C9ND mode shape is isolated and the stress-intensity factors are calculated from the energy release rates. The values presented in this figure are scaled to a unit value of far-field mid-ligament stress. Mode I is nearly constant and is dominant for crack lengths less than 0.3 in. (where most of the fatigue life is accumulated). The Mode III contributions increase with increasing crack length with the Mode III about equal to the Mode I component for crack lengths greater than 0.4 in. The Mode II component is insignificant.

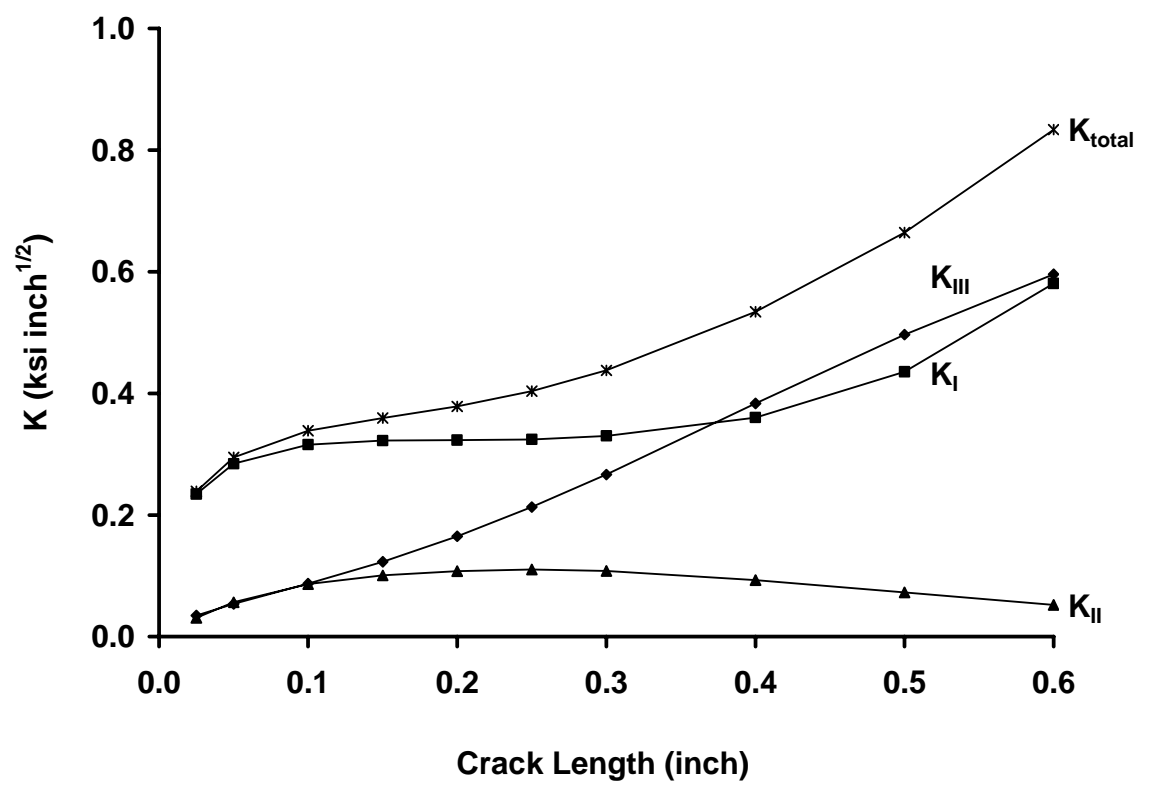

Figure 9. Stress-intensity Factors for the Shell-Dynamics Model for the C9ND mode - Upstream liner

The largest crack length considered in the shell-dynamic analysis for this comparison and for the life predictions is 0.6 in. because longer crack lengths exhibit a considerable Mode III component of the stress-intensity factor. The material data used to characterize crack growth behavior was derived from Mode I crack growth rate tests and is not necessarily appropriate for Mode III dominated crack growth. Most of the life is consumed while the crack is a corner crack (or short through-the-thickness crack), so stopping the life prediction at 0.6 in. is conservative and makes little difference to the overall calculated life. 
The analysis presented above for the upstream liner is repeated for the downstream liner. Recall that in the downstream liner there are three modes, 3ND, C4ND, and 5ND, that are active. The stress-intensity factors are calculated for various crack lengths using the shell-dynamics approach for the three modes and are presented in Figures 10 - 12.

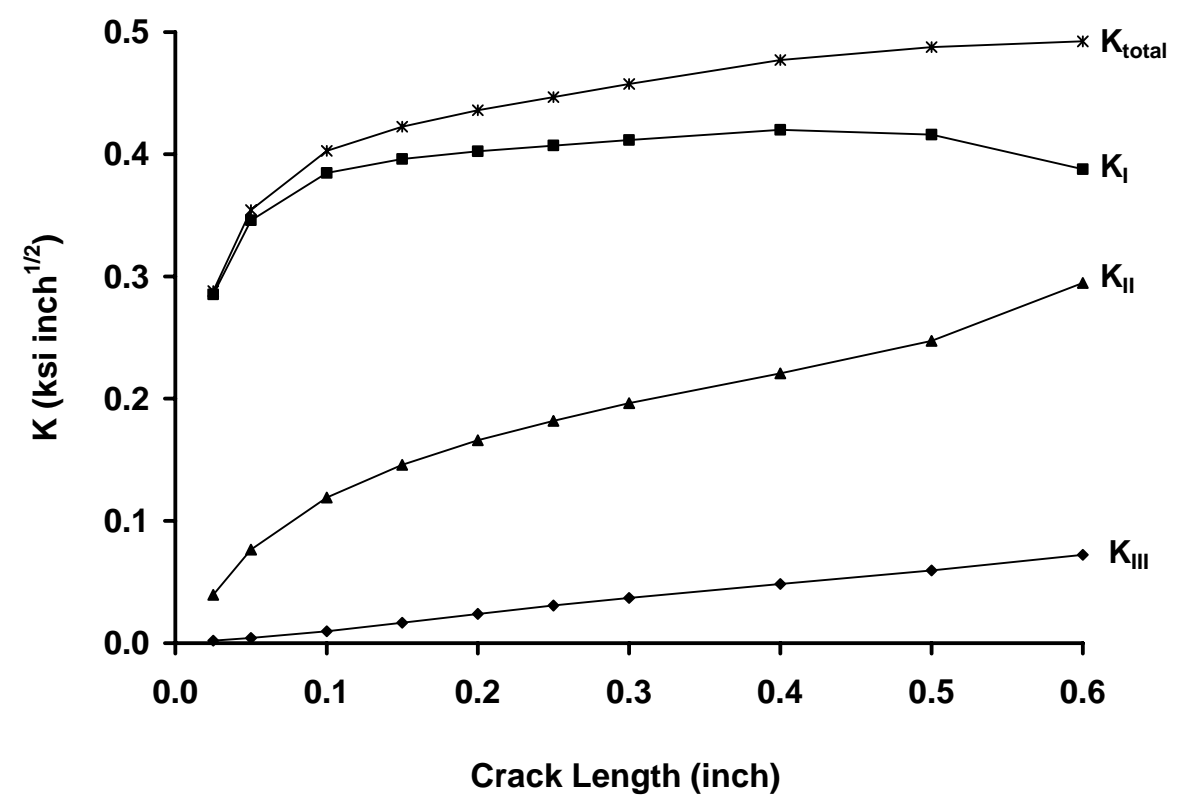

Figure 10. Stress-intensity Factors for the Shell-Dynamics Model for the 3ND mode - Downstream liner 


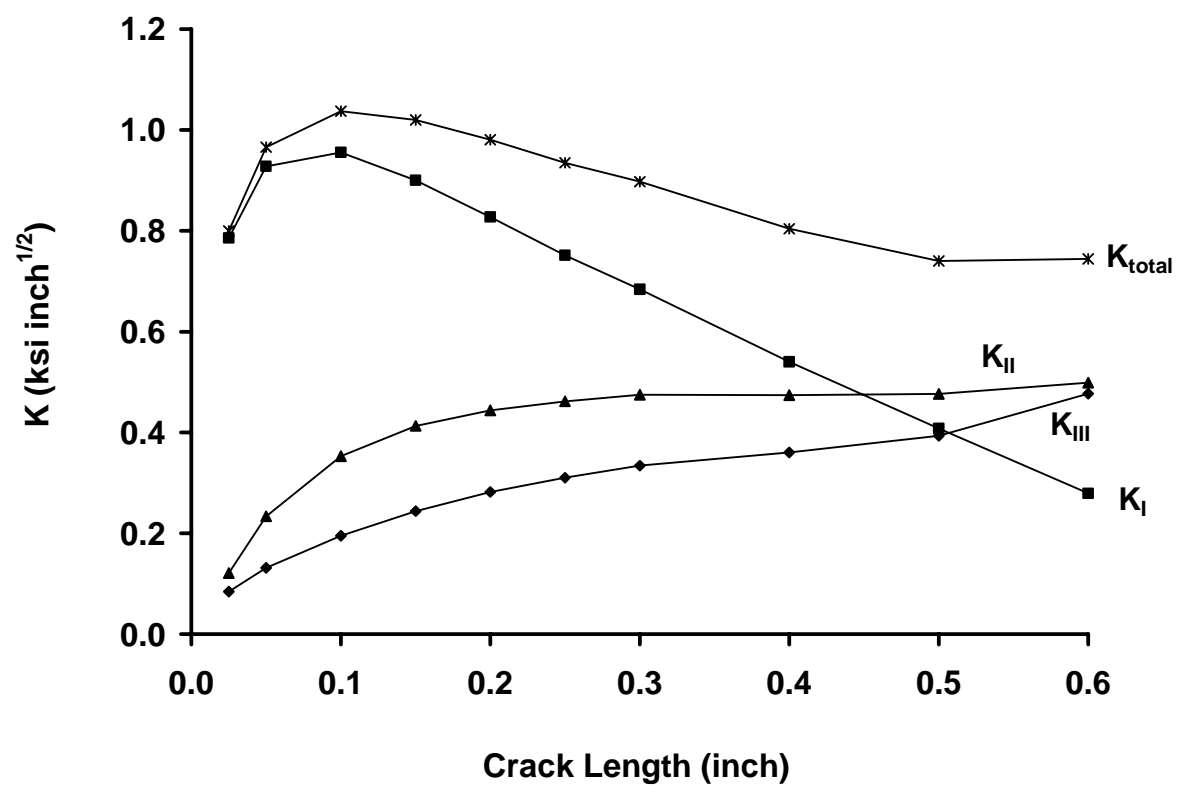

Figure 11. Stress-intensity Factors for the Shell-Dynamics Model for the C4ND mode - Downstream liner

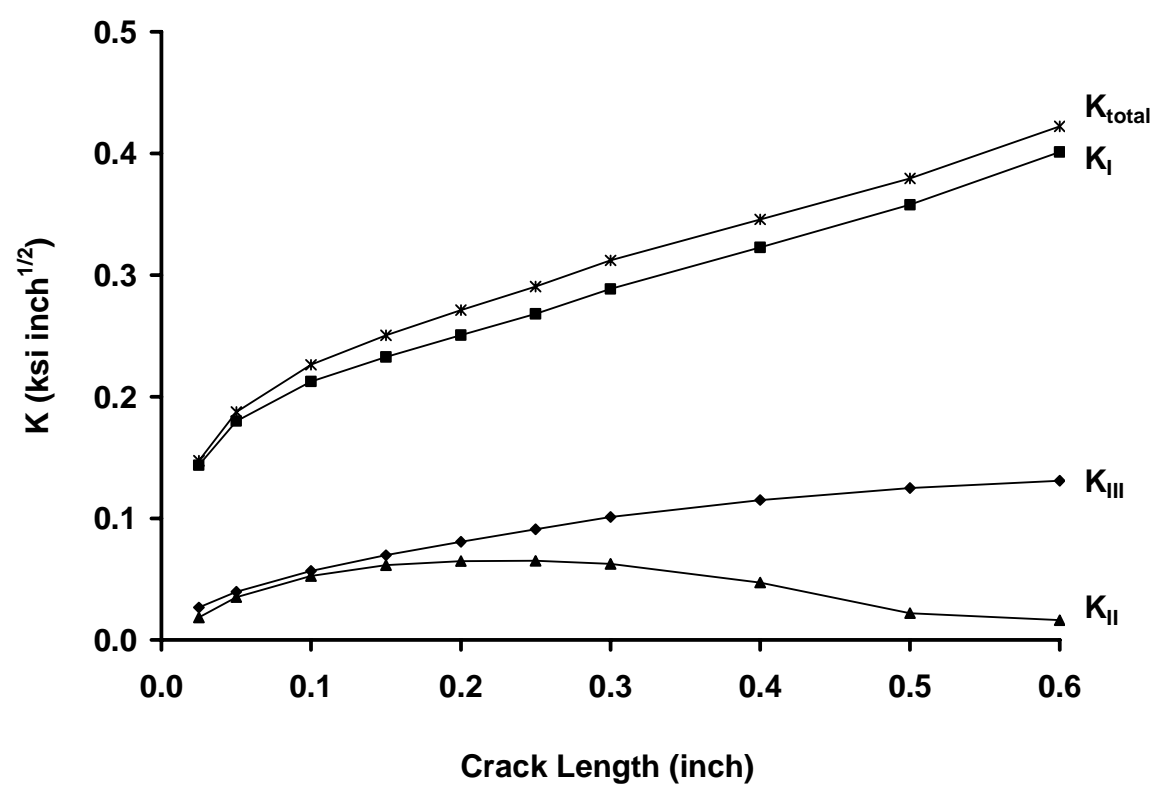

Figure 12. Stress-intensity Factors for the Shell-Dynamics Model for the 5ND mode - Downstream liner 
The stress-intensity factors for the four modes considered show wide ranging behavior in Figures 9 - 12. In all cases, when the crack is small (compared to the ligament), the stress-intensity factor is dominated by the Mode I value. However, as crack growth and load redistribution occur, each of the four modes responds differently. In all of the cases, the Mode I stress-intensity factor continues to contribute to a varying degree. The C9ND shape leads to near equal contributions of Modes I and III that monotonically increase, with an insignificant Mode II. In contrast, the total stress-intensity factor for the C4ND shape decreases after a peak value that occurs at about 0.1 in. of growth. These results indicate that modal deformations can lead to complex fracture mechanics behavior.

\section{Results}

The shell-dynamics $\mathrm{K}_{\text {total }}$ stress-intensity factors are used in the NASGRO calculations. The loading spectra are calculated as mid-ligament stresses and are used to scale the modal stressintensity factor results. These new results are then used to calculate fatigue lives for both liners at location B, as summarized in Table 1. An alternate procedure, termed the "transfer factor (TF) approach", is used to evaluate the life of the liners in Reference 1, and the results are included in this table for comparison. The transfer factor approach is very conservative and predicts lives of less than one flight.

Table 1. Predicted lives for upstream and downstream liners

(Crack assumed at location B, LHe crack growth data at $-423^{\circ} \mathrm{F}$ )

Upstream Liner

Life (flights)

\begin{tabular}{|c|c|c|}
\hline \hline $\begin{array}{c}\text { Initial Crack } \\
\text { Length, } \mathbf{c}_{\mathbf{i}} \text { (in) }\end{array}$ & $\begin{array}{c}\text { Shell-Dynamics } \\
\text { Approach }\end{array}$ & TF Approach \\
\hline 0.075 & 21 & 0.1 \\
\hline 0.02 & 39 & 0.3 \\
\hline
\end{tabular}

Downstream Liner

Life (flights)

\begin{tabular}{|c|c|c|}
\hline \hline $\begin{array}{c}\text { Initial Crack } \\
\text { Length, } \mathbf{c}_{\mathbf{i}} \text { (in) }\end{array}$ & $\begin{array}{c}\text { Shell Dynamics } \\
\text { Approach }\end{array}$ & TF Approach \\
\hline 0.075 & 0.2 & 0.1 \\
\hline 0.02 & 1.0 & 0.2 \\
\hline
\end{tabular}

As seen from this table, the shell-dynamic approach predicts longer life for the upstream liner compared to when the TF approach is used. The predicted fatigue life for the downstream liner is also longer using the shell-dynamics approach. However, even with an initial crack length as 
small as 0.02 in., failure of the downstream liner is predicted in about 1 flight using the shelldynamics approach. These results suggest that either (or both) the uncertainty in the load spectra leads to overly conservative life predictions or that the structure cannot tolerate cracks that can be found with traditional detection techniques. The flow liner cracking problem can be mitigated using any of the three following approaches:

1. Refine the load analysis to reduce the uncertainty in the load spectra, allowing the analysis to take advantage of the benefits of load sequence effects.

2. Refine the fatigue crack growth to account for the non-LEFM behavior of small cracks.

3. Develop inspection techniques that allow for the reliable detection of smaller fatigue cracks ( $<<0.02$ in.) and a process for eliminating the cracks from the structure.

The approach 3 above was chosen and the flow liner fatigue cracking issue was resolved by developing an inspection process that used a high resolution surface replication technique to detect cracks as small as 0.002 in. The process involved replicating a mold of the slot surface and examining the mold with a high magnification scanning electron microscope. The flow liners in the fleet of the three orbiters were examined (684 individual slots) and 50 cracks with lengths of $0.002 \mathrm{in}$. to $0.05 \mathrm{in}$. were detected. The texture of the slot surfaces was also examined and locations with the potential to initiate cracks (i.e. scratches and dents) were identified. The cracks and locations with surface damage were polished in an attempt to return the structure to a pristine condition. The flow liners were inspected with the surface replication technique after polishing and again after the Shuttle flight. No new cracks or additional surface damage were detected.

\section{Concluding Remarks}

A fracture mechanics-based study is performed for cracks detected in the flow liners in Space Shuttle Main Engines. The flow liners experience complex loading induced by complex flow and the resonance characteristics of the liner. A circumferential crack at the edge of the slot near the weld is considered because this location experiences the highest combined stresses. Fatigue loading spectra are developed by the loads and dynamics team, and these spectra are used to evaluate the life of the liners.

A shell-dynamics approach is used to simulate the dominant resonant frequencies experienced by the liner. The modes that correspond to these frequencies are prescribed on the shell, and the slot with the highest stress is identified. A circumferential crack is assumed to exist at the edge of this slot and near the weld. The stress-intensity factor for this crack is evaluated using the dominant mode shapes. The stress-intensity versus crack length for the liner is evaluated. This stressintensity factor-vs-crack length curve is used with the fatigue spectra to evaluate the life of the flow liners using NASGRO. While the upstream liner shows adequate life, the downstream liner results show that failure will occur within one flight for an initial crack length of 0.075 in. 
The flow liner fatigue cracking issue was resolved by developing an inspection process that used a high resolution surface replication technique to detect cracks as small as $0.002 \mathrm{in}$. The process involved making a mold of the slot surface and examining the mold with a high magnification scanning electron microscope. The detected cracks and locations with surface damage were polished to return the structure to a pristine condition. The flow liners were inspected with the surface replication technique after polishing and again after a Shuttle flight. The flow liners in the orbiter showed no new cracks or additional surface damage.

\section{References}

[1] Harris, C. E., et al., “Orbiter $\mathrm{LH}_{2}$ Feedline Flowliner Cracking Problem,” NASA/TM2005-213787/Version 1.0, NESC-RpP-04-11/04-004-E, July 2005.

[2] NASGRO Version 4.11 Manual, Southwest Research Institute, February 2004.

[3] Metallic Materials Properties Development and Standardization (MMPDS), DOT/FAA/AR-MMPDS-01, Federal Aviation Administration, Office of Aviation Research, Washington D.C., January 2003.

[4] Newman, Jr., J.C. and Raju, I.S., "Stress-intensity Factor Equations for Cracks in ThreeDimensional Finite Bodies Subjected to Tension and Bending Loads," Computational Methods in Mechanics of Fracture, S.N. Atluri, Ed., Elsevier, 1986, pp. 311-334.

[5] Rybicki, E.F. and Kanninen, M.F., "A Finite Element Calculation of Stress Intensity Factors by a Modified Crack Closure Integral,” Eng. Fracture Mech., Vol. 9, pp. 931938, 1977.

[6] Wang, J.T., Raju, I.S., Davila, G., and Sleight, D., “Computation of Strain Energy Release Rates for Skin-Stiffener Debonds Modeled with Plate Elements," $34^{\text {th }}$ AIAA SDM Conference, La Jolla, California (April 19-21, 1993). 